\title{
Lipoleiomyoma in Myomatous Uterus
}

\author{
Spasimir Todorov Shopov*
}

Department of Pathology at MBAL "Parvomai" Ltd, Parvomai, Bulgaria; Department of General and Clinical Pathology, Medical University - Plovdiv, Bulgaria

"Corresponding author: Shopov ST, Department of General and Clinical Pathology, Medical University - Plovdiv 15a Vassil Aprilov Blvd. 4000 Plovdiv, Bulgaria, Tel: 08786572561; E-mail: sshopov1@abv.bg

Received: May 23, 2019; Accepted: May 28, 2019; Published: June 05, 2019

\begin{abstract}
Lipoleiomyoma of the uterus is a rare benign tumor thought to be a variation of leiomyoma. The presence of fatty tissue in the myometrium is anomalous, interpreted as lipomatous degeneration, smooth muscle metaplasia or as a benign tumor called lipoleiomyoma. Most are found as accidental pathological findings after surgery. I report a case of lipoleiomyoma in a myomatous uterus in a 50-year-old woman who has had irregular vaginal bleeding.
\end{abstract}

Keywords: Lipoleiomyoma; Leiomioma; Uterus

\section{Introduction}

Uterine lipoleiomyoma, a benign tumor of the uterus, is rare, with the overall incidence reported between $0.03 \%$ and $0.2 \%$ [1], and comprising of $0.35 \%$ of uterine myomatous tumors [2].

It is mostly described as a variant of uterine leiomyoma with similar clinical course and presentation and is typically found in postmenopausal patients. Histologically, these tumors are composed of variable amounts of smooth muscle, fat cells, and fibrous tissue, developing likely secondary to fatty degeneration of smooth muscle cells of leiomyomas [3].

\section{Case Report}

A 50-year-old woman with irregular vaginal bleeding for 3 months.

Gynecological examination revealed no abnormalities of the vagina and vaginal portion of the cervix and no evident pathological change was detected with clinical examination. 
www.yumedtext.com | June-2019

Routine hematological investigations were within normal limits.

Findings of ultrasonography examination suggested bulky uterus with thickened endometrium of 3 mm and hyperechoic mass suggestive of myoma of posterior wall of the uterus, measuring $5.0 \mathrm{~cm}$ in diameter. In addition, ultrasonography revealed hyperechoic lesion of $4.5 \times 3.0 \mathrm{~cm}$ in the fundal region. Also, there were three subserosal leiomyomas of $0.5 \mathrm{~cm}$ to $1.0 \mathrm{~cm}$ in diameter each. Ovaries and fallopian tubes within a normal range.

The patient underwent total abdominal hysterectomy with bilateral salpingo-oophorectomy because of multiple leiomyomas. Operative treatment and the diagnosis were performed at the multiprofile hospital for active treatment "Parvomay" Ltd. No postsurgical complications were registered.

Macroscopically: uterus measured $14.0 \times 10.0 \times 6.5 \mathrm{~cm}$ and had five intramural and subserosal well-circumscribed round masses. The biggest nodule which was measuring $6.0 \mathrm{~cm}$ in diameter with a typical type for of uterine leiomyoma. The next largest intramouth junction $4.0 \times 3.0 \mathrm{~cm}$ has a grayish-yellowish cut surface and a soft consistency (FIG. 1). The other three leiomyomas, each with a diameter of $0.5 \mathrm{~cm}-1.0 \mathrm{~cm}$, show a rough-textured pattern with a white appearance on their surface. Cut a section of ovaries showed follicular cysts of $0.5 \mathrm{~cm}$ diameter each, corpus albicans and corpus luteum. The fallopian tubes appeared grossly normal.

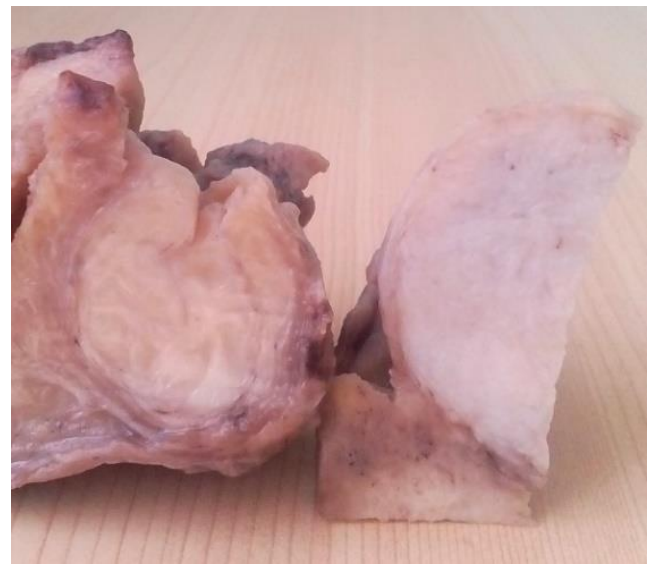

FIG. 1. Macroscopic picture: myomatous uterus - left lipoleiomyoma, right leiomyoma.

Histology Hematoxylin and Eosin (H\&E) (FIG. 2): Cervix with retention cysts. Corpus uteri: biggest nodule showed a mixture of bland, spindle-shaped smooth muscle cells without nuclear atypia and sections of hyalinosis (FIG. 2a). The second largest knot showed a mixture of smooth muscle fibers, the nuclei of which were extended with a finely dispersed chromatin and small nuclear cells between the hyaline fibers. Between these muscle cells, a significant amount of fat cells were visible. The adipose component was entirely mature without any lipoblasts (FIG. 2a, b \& c). Based on the above findings, the tumor was diagnosed as a benign lipoleiomyoma. Sections from the other nodules showed classical histomorphology of conventional uterine leiomyomata. The endometrium in an early secretory phase. Fallopian tubes have fibrosis. Sections from both the ovaries showed follicular cysts, corpus albicans, corpus luteum, and hemorrhagic corpus.

Diagnosis: Lipoleiomyoma in myomatous uterus. 


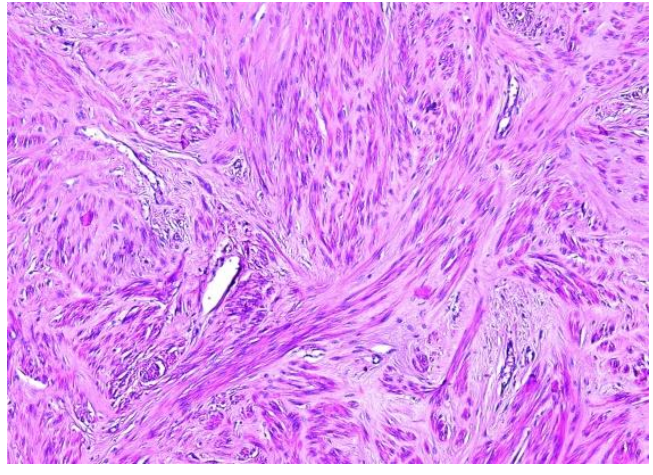

Fig. $2 \mathrm{a} \times 100$

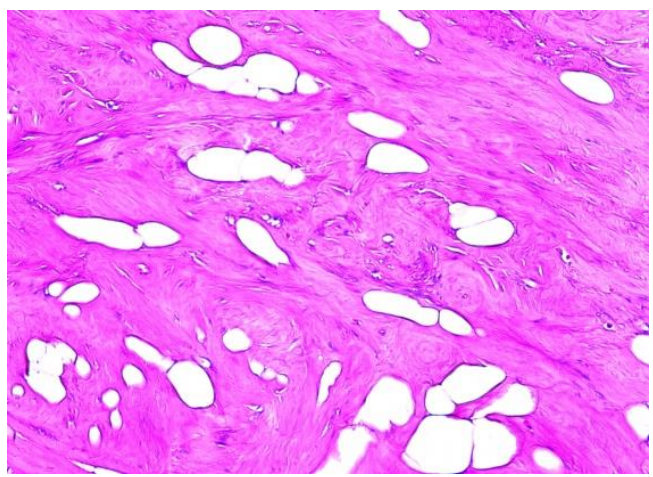

Fig. $2 \mathrm{c} \times 100$

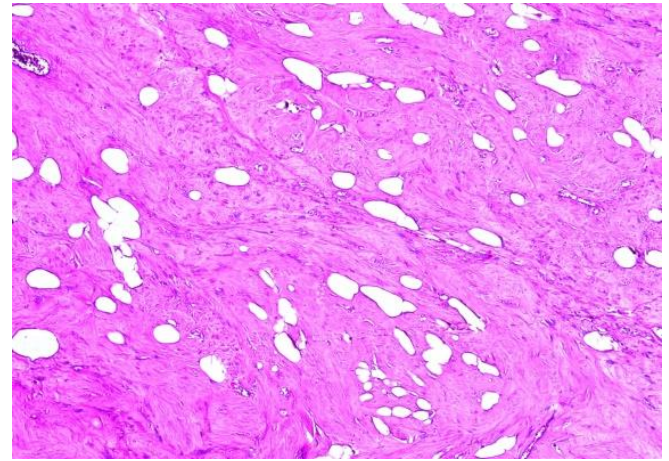

Fig. $2 b \times 50$

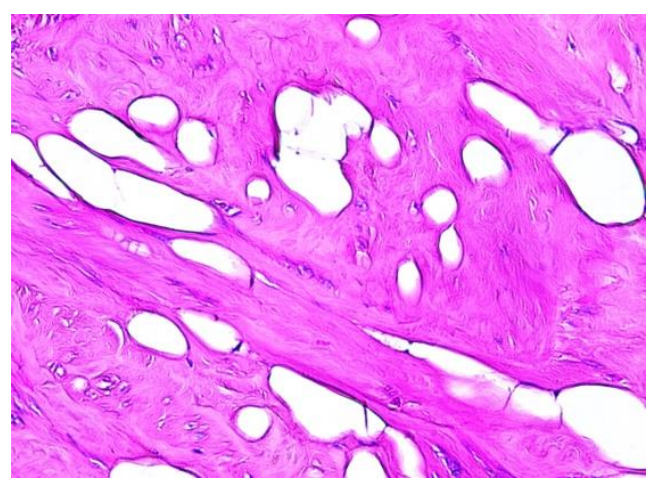

Fig. $2 d \times 200$

FIG. 2. Histology (H\&E): a. Bland smooth muscle cells are entrapped and isolated within fields of hyalinized stroma

(enlargement ×100); b. Smooth muscle cell proliferation admixed with mature adipocytes (enlargement ×50); $\mathrm{c}$.

Smooth muscle cell proliferation admixed with mature adipocytes (enlargement $\times 100$ ); $d$. Smooth muscle cell proliferation admixed with mature adipocytes (enlargement $\times 200)$.

\section{Discussion}

Lipoleiomyoma is an unusual tumor. These tumors showed characteristic histological findings, being composed of benign smooth muscle and mature adipose tissue. Similar tumors in the uterus are known as lipoleiomyomas. Lipoleiomyomas occur in different locations including cervix and ovaries [4]. Pathogenetically, it is assumed they are said to be subsets of leiomyomas with fatty degeneration of smooth muscle cells into adipose tissue [1]. Supposed that these tumors may arise due to misplaced remnants of fatty cells of the embryo, due to the extension of retroperitoneal or peritoneal fat perivascularly, due to fatty infiltration of the connective tissue, or due to the transformation of the mesenchymal totipotent cell [5]. The estrogenic state may also be a contributing factor. The change in lipid metabolism during menopause may play a role in its development. Furthermore, these patients commonly suffer from metabolic disorders such as hypothyroidism, hyperlipidemia, and diabetes mellitus [1]. They are often seen in postmenopausal women who remain either asymptomatic or present with symptoms similar to those of uterine leiomyomas. They present usually as solitary enlarged masses of varying sizes with the common site of occurrence in the corpus of the uterus [1,5]. Recognition of this rare and benign tumor is of great importance, because it may be confused with other uterine tumors: spindle cell lipoma, angiolipoma, angiomyolipoma, 
www.yumedtext.com | June-2019

atypical lipoma, myelolipoma, myxoid mesenchymal tumors, well-differentiated liposarcoma, malignant mixed Müllerian tumor with heterologous liposarcomatous differentiation, ovarian fatty tumors, and benign or malignant degeneration of ordinary leiomyomas [5]. Uterine lipoleiomyomas are often diagnosed preoperatively as uterine leiomyomas. Ultrasonography is specific enough to allow for a description of tissues and an accurate preoperative diagnosis. Computed tomography or magnetic resonance imaging can exclusively show the fat content within the tumor [1]. Therefore, the combined study would benefit from preoperative diagnosis. However, the final diagnosis is histological. To the best of our knowledge, this is the first reported case of lipoleiomyoma in myomatous uteri in Bulgaria. What is important in this case is the rare macroscopic photo showing the obvious difference between leiomyoma and lipoleiomyoma.

\section{Conclusion}

Uterine lipoleiomyomas are benign uterine lesions that, accounted for small \% of benign uterine leiomyomas. They are a variant of leiomyoma and showed a favorable flowing. However, the pathogenesis and clinical significance of these rare neoplasms remain to be clarified.

\section{REFERENCES}

1. Nazir HM, Mehta S, Seena CR, et al. Uterine Lipoleiomyoma: A Report of Two Cases. J Clin Imaging Sci. 2017;7:26.

2. Aung T, Goto M, Nomoto M, et al. Uterine lipoleiomyoma: a histopathological review of 17 cases. Pathol Int. 2004;54(10):751-8.

3. Avritscher R, Iyer RB, Ro J, et al. Lipoleiomyoma of the uterus. AJR Am J Roentgenol. 2001;177(4):856.

4. Kumar S, Garg S, Rana P, et al. Lipoleiomyoma of uterus: uncommon incidental finding. Gynecol Obstet. 2013;3(03):145.

5. Akbulut M, Gündoğan M, Yörükoğlu A. Clinical and pathological features of lipoleiomyoma of the uterine corpus: a review of 76 cases. Balkan Med J. 2014;31(3):224-9. 